\title{
A novel 3D intestine barrier model to study the immune response upon exposure to microplastics
}

\author{
Roman Lehner $^{1} \cdot$ Wendel Wohlleben $^{2} \cdot$ Dedy Septiadi $^{1} \cdot$ Robert Landsiedel $^{3} \cdot$ Alke Petri-Fink $^{1}$. \\ Barbara Rothen-Rutishauser ${ }^{1}$
}

\begin{abstract}
The plausibility of human exposure to microplastics has increased within the last years. Microplastics have been found in different food types including seafood, salt, sugar and beverages. So far, human health effects of microplastics after ingestion are unknown. Herein, we designed a novel, three-dimensional in vitro intestinal model consisting of the human intestinal epithelial cell lines Caco-2 and HT29-MTX-E12 as well as human blood monocyte-derived macrophages and dendritic cells that is suitable to assess the possible effects of ingested microplastics. Relevant microplastic particles (in the order of 50-500 $\mu \mathrm{m}$ ), including polymers representing tire wear and polyolefins, which represent major sources of microplastic in the EU, were compared to other polymer classes and an inorganic microparticle, healing earth, which is intended for human consumption. Microplastic particles were exposed at concentrations of $823.5-1380.0 \mu \mathrm{g} / \mathrm{cm}^{2}$ to the model using a dry pow-der insufflator system to aerosolize the particles directly on the intestinal model's surface. Cytotoxicity was investigated after 6,24 and $48 \mathrm{~h}$ of exposure via measuring the release of lactate dehydrogenase. Inflammatory end points including the cytokines IL-8, TNF $\alpha$ and IL-1 $\beta$ as well as changes of the barrier integrity after exposure were additionally monitored. We demonstrated that all of the microplastics and the healing earth particles did not cause any significant cytotoxicity or release of (pro-)inflammatory cytokines and did not change the barrier integrity of the co-culture at any of the time points investigated.
\end{abstract}

Keywords Microplastics · Polymers $\cdot$ Tire dust $\cdot$ Polyamide $\cdot$ Polyurethane $\cdot$ Polypropylene $\cdot$ Human hazard $\cdot$ 3D intestine co-culture model $\cdot($ Pro-)inflammatory cytokines

\section{Introduction}

Plastics have shaped our society within the last centuries like no other material has. Their production has increased to more than 335 million metric tons worldwide in 2016 with around $40 \%$ of all plastics being incorporated into single use

Roman Lehner

roman.lehner@unifr.ch

1 Adolphe Merkle Institute, University of Fribourg, Chemin des Verdiers 4, 1700 Fribourg, Switzerland

2 BASF SE, Advanced Materials Research, 67056 Ludwigshafen, Germany

3 Experimental Toxicology and Ecology, GV/TB, Z470, BASF SE, Carl-Bosch-Str. 38, 67056 Ludwigshafen, Germany products such as plastic bags, food and drink containers, or cups (Plastic Europe 2018). The growing production of synthetic plastic products has also led to a substantial increase of poorly managed plastic waste, which results in their widespread and increasing accumulation within the environment.

A combination of chemical and physical processes involving the exposure to ultraviolet portion of sunlight in combination with mechanical action can cause the material to become brittle and degrade into smaller sized pieces (Eubeler et al. 2010; Yousif and Haddad 2013). Plastic particles at sizes $<5 \mathrm{~mm}$ are referred to as microplastics (MPs) and can be categorized into primary and secondary particles (Rezania et al. 2018). Primary MPs are intentionally manufactured for industrial purposes, e.g., pellets as precursors to other products, or for direct use as abrasives in cleaning products such as facial scrubs (Fendall and Sewell 2009; Cole et al. 2011). Secondary MPs are derived from the degradation of plastic litter by continuous weathering processes, both land and sea, and are dependent on the polymer type 
and morphology (Ryan et al. 2009). The main types of secondary microplastics observed in the environment include particles of polypropylene (PP, a polyolefine), tire dust, and polyamide (PA), which are environmentally relevant because nylon is the most common constituent of fishing nets that can degrade during use and/or when abandoned. In addition to these particle types, polyurethanes (PU), which are not frequently found in either freshwater or marine environments but are a versatile class of engineering polymers that can be adapted to various uses (e.g., for shoe soles), may generate relevant wear particles (Wu et al. 2017).

The plausibility of human exposure to MPs has increased within the last years. Several studies have shown that MPs can be found in seafood (Van Cauwenberghe and Janssen 2014; Rochman et al. 2015; Li et al. 2015), along with various convenience foods and beverages such as sugar (Liebezeit and Liebezeit 2013), salt (Iñiguez et al. 2017), beer (Liebezeit and Liebezeit 2014), and tap (Kosuth et al. 2018) and bottled water (Mason et al. 2018). In addition, the release of fibers from drying synthetic clothes, wind-driven transport from MPs found in sludge, as well as atmospheric fallout of particles poses a risk of exposure through inhalation for humans (Bouwmeester et al. 2015; Prata 2018). The presence of MPs in the atmosphere and their subsequent potential to undergo fallout, resulting in contamination of ecosystems, have already been shown (Allen et al. 2019). However, ingestion of MP particles is likely to represent the main route of exposure in humans, since MP particles can be ingested by eating contaminated seafood or drinking water (World Health Organization 2019). This makes the gastrointestinal tract (GIT) the most likely primary exposure site. Currently, the knowledge about exposure levels and possible hazardous effects of MPs on human health is limited (Wright and Kelly 2017).

Within the last decades, tremendous effort has been put into the development of realistic and predictive $3 \mathrm{D}$ tissue models mimicking the human intestinal tract to investigate the hazard of ingested materials. Complex multicellular in vitro co-cultures differ morphologically and physiologically from simple single cell models and allow researchers to more closely mimic the in situ structure and function. In addition, they better replicate responses to external stimuli compared to single cell models and allow specific end points such as the release of (pro-)inflammatory cytokines or the production of reactive oxygen species to be studied (Zietarska et al. 2007; Lee et al. 2008; Edmondson et al. 2014). Numerous 3D human intestinal models have been developed to mimic the human intestinal barrier, including the mucosa layer, by combining Caco-2 enterocytes and mucus-secreting goblet cells, HT29 and HT29-MTX, as well as specialized epithelial M cells (Hilgendorf et al. 2000; Schimpel et al. 2014; Béduneau et al. 2014). Most of the studies published applying the Caco-2/HT29 co-culture model focus on studying ingested material or drug permeability/transport (Antunes et al. 2013; Araújo and Sarmento 2013). In addition, few studies attempt to mimic pathophysiological changes occurring in vivo in the inflamed intestine by introducing immune cells such as macrophages and/or dendritic cells into their model (Leonard et al. 2010; Susewind et al. 2016; Kämpfer et al. 2017).

Herein, we designed a novel human in vitro intestinal model consisting of the human intestinal epithelial cell lines Caco-2 and HT29-MTX as well as both immune cell types, human blood monocyte-derived macrophages (MDMs) and dendritic cells (MDDCs), to assess the possible effects of ingested microplastics. We used environmentally relevant microplastic particles, including polymers representing tire wear (Kole et al. 2017) and polyolefins, e.g., particles of polypropylene (PP), which represent major sources of microplastic in the EU, which we compared against other polymer classes, e.g., polyamide (PA) and harder cross-linked or softer thermoplastic version of polyurethanes (PU). The scope of comparing different microplastics is complemented by an inorganic microparticle, which consists of aluminosilicates, that is intended for human consumption (i.e., healing earth). The intentionally micronized fine powders are thought to represent the lowest particle size that can be generated by mechanical forces during use and degradation ("secondary microplastic"), but in fact two of the fine powders are commercially traded and thus examples of "primary microplastic". The material was aerosolized with an insufflator system directly onto the cell surface. This approach allows for the determination of the concentration of particles interacting with the cells. Cytotoxicity, cell morphology, barrier characteristics, and inflammatory endpoints were investigated after 6,24 and 48 h exposure time.

\section{Materials and methods}

\section{Particle micronization}

The approach to provide microplastic particles in kilogram quantities mimicking actual released materials from polymer parts or tires was developed by the SUN project (Nowack et al. 2016). In short, the polymer pellets of PP_Sun, PA6 and TPU_ester were frozen to cryogenic temperatures (at $-193{ }^{\circ} \mathrm{C}$, liquid $\mathrm{N}_{2}$ ) to maximize solidity and brittleness, then micronized in a mill (impeller breaker, Pallmann PPL $18)$, with the impeller rotated at $10,000 \mathrm{rpm}(92 \mathrm{~m} / \mathrm{s}$ circumferential speed). Within $40 \mathrm{~min}$, the total amount of $1 \mathrm{~kg}$ of the granulates was passed through the $0.3 \mathrm{~mm}$ gap between the rotor/stator impeller. The PU_hardened was cast in cmsized plates, then manually broken before cryo-milling. Note that the cryogenic temperature does not change the polymer chains, and the effect of brittleness is fully reversible. In 
SUN terminology, the microplastics resulting from cryomilling are "fragmented products" (FP), and the fraction that we study here are "sieved fragmented products" (SFP) (Nowack et al. 2016). Sieving was performed manually on all materials with a nominal cutoff of $0.3 \mathrm{~mm}$ to remove particles above $300 \mu \mathrm{m}$ that are rare in number, but too relevant to ignore in mass-based dose metrics. The healing earth and truck tire rubber were provided in micronized form, but the tire powder was produced by a similar cryo-milling, according to the recycling company.

\section{Particle characterization}

Laser diffraction: FP were dispersed in a concentration of $1 \mathrm{~g} / \mathrm{l}$ by sonication in water containing $0.5 \mathrm{~g} / \mathrm{l} \mathrm{SDS}$ (sodium dodecylsulfate) and were characterized by laser diffraction (Malvern Mastersizer 3000). Scanning electron microscopy (SEM): samples were fixed on an adhesive film, coated with $9 \mathrm{~nm}$ Pt and investigated on a JSM 7500TFE (Jeol Company) operated at $5 \mathrm{keV}$. The topographic images were taken with secondary electrons (SE). Secondary ion mass spectrometry (SIMS): samples were powdered onto double-stick tape, and carefully tossed to remove lose particles. The primary ion source was $\mathrm{Bi}^{3+}$ at $25 \mathrm{keV}$; the secondary ions were detected at positive and negative polarity. The signal was integrated across an area of $250 \times 250 \mu \mathrm{m}^{2}$.

\section{Cell cultures}

The human colon (colorectal adenocarcinoma) cell line Caco-2 was obtained from American Type Culture Collection (ATCC, HTB-37) and the human colon cell line HT29-MTX-E12 was obtained from European Collection of Authenticated Cell Cultures (ECACC). Both cell types were cultivated at $37{ }^{\circ} \mathrm{C}$ under a $5 \% \mathrm{CO}_{2}$ water saturated atmosphere in complete medium consisting of Dulbecco's modified Eagle medium (DMEM) (Gibco) supplemented with $10 \%$ non-heat inactivated fetal bovine serum (Gibco), $1 \%$ MEM nonessential amino acids (Gibco), 1\% L-glutamine (Gibco), and $1 \%$ penicillin and streptomycin (Gibco). Routinely, Caco-2 and HT29-MTX cells were grown in $75 \mathrm{~cm}^{2}$ flasks (Corning, USA) and sub-cultured twice per week at a ratio of $1: 4$ and $1: 6$, respectively.

Human blood monocytes were isolated from buffy coats provided by the blood donation service SRK Bern as previously described by Lehmann et al. (2011) and purified using CD14 magnetic beads (Microbeads, Milteny Biotech, Germany). Monocytes were cultured at a density of $10^{6}$ cells/ $\mathrm{ml}$ in Roswell Park Memorial Institute medium (RPMI 1640) supplemented with $10 \%$ fetal bovine serum (FBS), $1 \%$ L-glutamine, and $1 \%$ penicillin/streptomycin. For cell differentiation into MDDC and MDM, GM-CSF (10 ng/ml) and interleukin 4 (IL-4) $(10 \mathrm{ng} / \mathrm{ml})$ resp. M-CSF (10 ng/ml) were added for 7 days. MDDC and MDM from one donor were used for one independent data set.

\section{D co-culture model}

For the design and optimization of the co-cultures, monocultures of Caco-2 and HT29-MTX cells as well as co-cultures of Caco-2/HT29-MTX at a ratio of 9:1 were grown on 12-well Transwell ${ }^{\mathrm{TM}}$ inserts (Corning Incorporated, USA; $3 \mu \mathrm{m}$ mean pore size, $0.9 \mathrm{~cm}^{2}$ surface area) placed in 12-well wells. Mono- and co-cultures were seeded on the apical chamber of the inserts at a final density of $2.2 \times 10^{5}$ cells/ $\mathrm{cm}^{2}$. Cells were grown for 20 days with medium changes every other day for proper brush boarder formation (Pinto et al. 1983). On day 20, the co-cultures were assembled by adding MDMs and MDDCs to the basolateral side. Briefly, the inserts were placed in a sterile Petri dish turned upside down, and the cells at the bottom of the membrane which have grown through the pores were gently abraded with a cell scraper. A mixture of MDDC $\left(5 \times 10^{4}\right)$ and MDM $\left(1.2 \times 10^{4}\right)$ cells per 12 -well insert were pipetted onto the bottom side of the inserts and incubated for $70 \mathrm{~min}$ at $37^{\circ} \mathrm{C}$ and $5 \% \mathrm{CO}_{2}$. Afterwards, the inserts were placed back into the well plate containing $1.5 \mathrm{ml}$ of preheated fresh supplemented DMEM medium and the co-cultures were incubated for an additional 24 h, i.e. day 21.

\section{Visualization of the cells with laser scanning microscopy}

For the visualization of the individual cells, Caco-2, MDM and MDDC cells were stained using different fluorophores (Vybrant ${ }^{\circledR}$ multicolor cell labeling kit, Invitrogen Molecular Probes) prior to the co-culture assembly. Briefly, MDM or MDDC cells were scrapped, centrifuged, resuspended in $200 \mu \mathrm{l}$ of RPMI 1640 and treated with vibrant dye DiI or $\mathrm{DiO}(1: 200)$ for $30 \mathrm{~min}$ at $37{ }^{\circ} \mathrm{C}$ and $5 \% \mathrm{CO}_{2}$. Afterward, cells were centrifuged and washed three times with PBS to remove excess dye. The monolayer of Caco-2 cells was treated with vibrant dye DiD (1:200) and incubated for $30 \mathrm{~min}$ at $37{ }^{\circ} \mathrm{C}$ and $5 \% \mathrm{CO}_{2}$. Subsequently, cells were washed three times with PBS and the co-culture was set up as described above. Image processing was performed using the restoration software IMARIS (Bitplane AG, Switzerland).

\section{Morphological characterization of the co-culture model}

To study the development of the microvilli brush border by enterocytes over time, scanning electron microscopy imaging was conducted. Cells at day 2 and 14 were washed twice with PBS and fixed in Karnovsky fixative $(2.5 \%$ 
glutaraldehyde, $2 \%$ paraformaldehyde) for $2 \mathrm{~h}$. Subsequent dehydration was carried out through a graded series of ethanol $(20-100 \%)$ following a standard procedure. Filter membranes were cut out and mounted on a sample holder using carbon black tapes. Samples were dried by putting them into an exicator for $24 \mathrm{~h}$ and sputtered with gold. Images were acquired using a scanning electron microscope (TESCAN Mira 3 LM field emission).

\section{Transepithelial electrical resistance (TEER)}

The integrity of the Caco-2 monolayer and the Caco-2/ HT29-MTX co-culture was monitored by TEER measurements using the Millicell Electrical Resistance System (Millicell ${ }^{\circledR}$ ERS-2, EMD Millipore Corporation, MA, USA). Cells grown on inserts were washed with PBS and TEER measurements were conducted in triplicates in three defined spots per insert. Only mono- and co-culture layers with TEER values higher than $200 \Omega \mathrm{cm}^{2}$ after day 21 were used in experiments.

\section{Cell layer integrity}

Cell layer integrity of the Caco-2 monolayer and the Caco-2/ HT29-MTX co-culture was evaluated using fluorescein isothiocyanate (FITC)-dextran $4 \mathrm{kDa}$ (Sigma Aldrich) and fluorescein $330 \mathrm{Da}$ (Sigma Aldrich) in the presence and absence of $2.5 \mathrm{mM}$ EDTA (Sigma Aldrich). EDTA is known to disrupt tight junctions via its $\mathrm{Ca}^{2+}$ chelating effect and activation of protein kinase $\mathrm{C}$. Transport of the dye was assessed after day 21 from apical to basolateral direction. Positive controls were pre-incubated with EDTA solution in HBSS apical and basolateral for $60 \mathrm{~min}$. Inserts were washed with HBSS and exposed to $1 \mathrm{mg} / \mathrm{ml}$ FITC-dextran $4 \mathrm{kDa}$ and fluorescein in HBSS for $60 \mathrm{~min}$. Samples from the apical and basolateral part were collected and the fluorescent signal was determined by the microplate reader (Tristar LB 941, Berthold Technologies; using the following filters setup: $\lambda$ ex $/ \lambda \mathrm{em}: 485 / 535 \mathrm{~nm}$ ). The results are presented as percentage of compound in the basolateral chamber in comparison to the total amount added.

\section{Mucus layer staining}

The production of mucus was assessed in Caco- 2 and HT29MTX monocultures as well as Caco-2/HT29-MTX co-cultures at a ratio of 9:1. Cells were fixed with cold methacarn solution (60\% methanol, $30 \%$ chloroform, $10 \%$ acetic acid) for $1 \mathrm{~h}$ at $4{ }^{\circ} \mathrm{C}$. Methacarn allows the preservation of the mucus layer. Cells were washed with tap water and 3\% acetic acid was added for $3 \mathrm{~min}$. Subsequently, $1 \%$ Alcian blue solution was added in the apical compartment for $20 \mathrm{~min}$ at room temperature. Multiple washes with MilliQ water were used to remove excess Alcian blue prior to imaging with a bright field microscope (Motic AE2000). Mucus production and identification of goblet cells within the co-culture was also determined by histochemical analysis. Briefly, cell samples were fixed in Methacarn solution for $1 \mathrm{~h}$, dehydrated, and embedded in paraffin prior to sectioning with a microtome. Samples were pathologically evaluated using hematoxylin/eosin and Alcian blue staining. Mucus production was further assessed with confocal microscopy. Cell samples were fixed in Methacarn solution for $1 \mathrm{~h}$, subsequently washed with PBS and permeabilized using $0.2 \%$ Triton-X-100 in PBS. Afterward, cells were blocked using $3 \%$ goat serum for $30 \mathrm{~min}$ and then incubated with the primary antibody MUC2 rabbit (NBP1-31231, Novusbio) overnight at $4{ }^{\circ} \mathrm{C}$. On the next day, cells were washed three times with PBS and incubated with the secondary antibody as well as DAPI (Sigma-Aldrich) for the cell nucleus staining for $1 \mathrm{~h}$. After additional washes, cells were mounted on glass slides and observed using an inverted laser scanning confocal microscope (LSM 710, Zeiss, Germany).

\section{MP exposure}

Prior to the exposure of the materials, the medium in the apical compartments of the cultures was removed. All the materials were nebulized directly onto the cells using a dry powder insufflator (Model DP-4; PennCentury Inc., Philadelphia, USA) connected to a nebulization chamber. The materials were loaded into a sample chamber and then pushed through the device by administering air using a $10 \mathrm{ml}$ commercial syringe. For optimal exposure of the materials, and to avoid a release of the aerosols into the environment, the insufflator was connected to a closed nebulization chamber placed over the inserts. The quantification of the exposed material was done by balancing the insufflator sample chamber before and after the exposure. Each material was tested in triplicates.

\section{Cell viability assay}

The effects of the different materials on the viability of the cells from the 3D co-culture model were investigated by the release of lactate dehydrogenase (LDH) into the supernatant as a result of cell membrane rupture. LDH release was evaluated using a commercially available LDH diagnostic kit (Roche Applied Science, Germany), according to the manufacturer's protocol. Each material was tested in triplicate and the enzyme activity was measured photometrically at an absorbance of $490 \mathrm{~nm}$ with reference wavelength of $630 \mathrm{~nm}$. As a positive control $0.2 \%$ Triton-X-100 was applied apically. LDH values are presented relative to the negative control (untreated cells). 
The (pro-)inflammatory response of the co-cultures after exposure of the materials for $24 \mathrm{~h}$ was investigated by quantifying the release of interleukin- 8 (IL-8), interleukin- $1 \beta$ (IL-1 $\beta$ ) and tumor necrosis factor $\alpha(\mathrm{TNF} \alpha)$ into the basal cell culture well. The ELISA was run using the commercially available DuoSet ELISA Development Kit (R\&D Systems, Switzerland) according to the supplier's protocol. Lipopolysaccharide (LPS, from E. coli at $10 \mu \mathrm{g} / \mathrm{ml}$ ) served as the positive control for the (pro-)inflammatory response for all cytokines. The concentrations were determined spectrophotometrically at $450 \mathrm{~nm}$ using a microplate reader (BioRad, Switzerland). Analyses were conducted in triplicate for each replicate.

\section{Tight junction formation}

The formation of tight junctions after day 21 was investigated using the primary antibody ZO-1 rabbit (Invitrogen) and the secondary antibody Alexa Fluor 488 goat anti-rabbit.

Briefly, mono- and co-cultures were washed after day 21 with PBS. Cells were fixed using 4\% paraformaldehyde for 15 min, subsequently washed with PBS and permeabilized using $0.2 \%$ Triton-X-100 in PBS. Afterward, cells were blocked using 1\% BSA and $0.3 \mathrm{M}$ glycine in PBS for $30 \mathrm{~min}$ and then incubated with the primary antibody $\mathrm{ZO}-1$ rabbit overnight at $4{ }^{\circ} \mathrm{C}$. On the next day, cells were washed three times with PBS and incubated with the secondary antibody as well as DAPI (Sigma-Aldrich) for the cell nucleus staining for $1 \mathrm{~h}$. After additional washings, cells were mounted on glass slides and observed using an inverted laser scanning confocal microscope (LSM 710, Zeiss, Germany).

\section{Immunofluorescence staining of MDMs and MDDCs}

Briefly, co-cultures were washed after day 21 with PBS. Cells were fixed using $4 \%$ paraformaldehyde for $15 \mathrm{~min}$, subsequently washed with PBS and permeabilized using $0.2 \%$ Triton-X-100 in PBS. Afterward, cells were blocked using 1\% BSA and 0.3 M glycine in PBS for $30 \mathrm{~min}$ and then incubated with the primary antibodies rabbit anti-CD83 and mouse anti-CD68 at room temperature for $2 \mathrm{~h}$. Cells were washed three times with PBS and incubated with the secondary antibodies as well as DAPI (Sigma-Aldrich) for the cell nucleus and Rhodamine (Sigma-Aldrich) for the actin filament staining for $1 \mathrm{~h}$. After additional washings, cells were mounted on glass slides and observed using an inverted laser scanning confocal microscope (LSM 710, Zeiss, Germany). Image processing was performed using the restoration software IMARIS (Bitplane AG, Switzerland).
All data are presented as mean \pm standard deviation. A total of three independent experiments $(n=3)$ were performed for the exposure measurements, cell viability, TEER, and (pro-)inflammatory cell responses. Statistical analysis was performed using GraphPad Prism (GraphPad Software Inc., La Jolla, USA). Assuming normal distribution of the data sets, a parametric one-way analysis of variance (ANOVA) was performed, followed by Dunnett's multiple comparison test. In all experiments, results were considered significant if $p<0.05$.

\section{Results}

\section{Microparticle characterization and relation to polymer uses}

All polymers were micronized by cryogenic milling and sieving to obtain fine powders (Pallmann PPL 18) (Nowack et al. 2016). In Fig. 1a, tire rubber particles are shown and provide an example of the irregularity of the final material.

Here, we sourced from MRH (Mülsener Rohstoff- und Handelsgesellschaft mbH, 08132 Mülsen) the finest available size fraction ("Gummimehl Type K 0002"). At higher magnification, submicron structures are visible on the surface of particles, and are attributed to the embedded fillers (Fig. 1b). The size distribution has no relevant fractions below $10 \mu \mathrm{m}$, and a median of $\mathrm{D} 50=72 \mu \mathrm{m}$ (Fig. 1c). The surface chemistry of the rubber particles is rather clean in SIMS spectra (Fig. 1d), because natural rubber and carbon black do not have characteristic spectra. The anion spectrum shows traces of fatty acids that are identified as surface adsorbates because they are easily removed by ion sputtering.

The particle size distributions of all other materials (Figure SI_1) are summarized by Table 1 . The median values (D50) range from 72 to $282 \mu \mathrm{m}$. The morphology of all materials is documented in Figures SI_2 to SI_7. The surface of neat PA6 particles (Figure SI_2) does not reveal additives, but bears traces of surfactant (SIMS data not shown). The PP is a thermoplastic grade that is intended for injection molding of, e.g., automotive parts and contains antioxidant stabilizers. The specific micronized form was previously studied for their physicochemical properties and ecotoxicological effects in the SUN project (Amorim et al. 2018). Further characterization showed a surface chemistry composed of $99.2 \%$ carbon atoms, and $0.8 \%$ oxygen, resulting in a hydrophobic surface (Amorim et al. 2018).

The TPU studied here was synthesized using a polyesterol, and thus has the ester bond in the polymer backbone. On the surface of the particles, some Acrawax additive is 

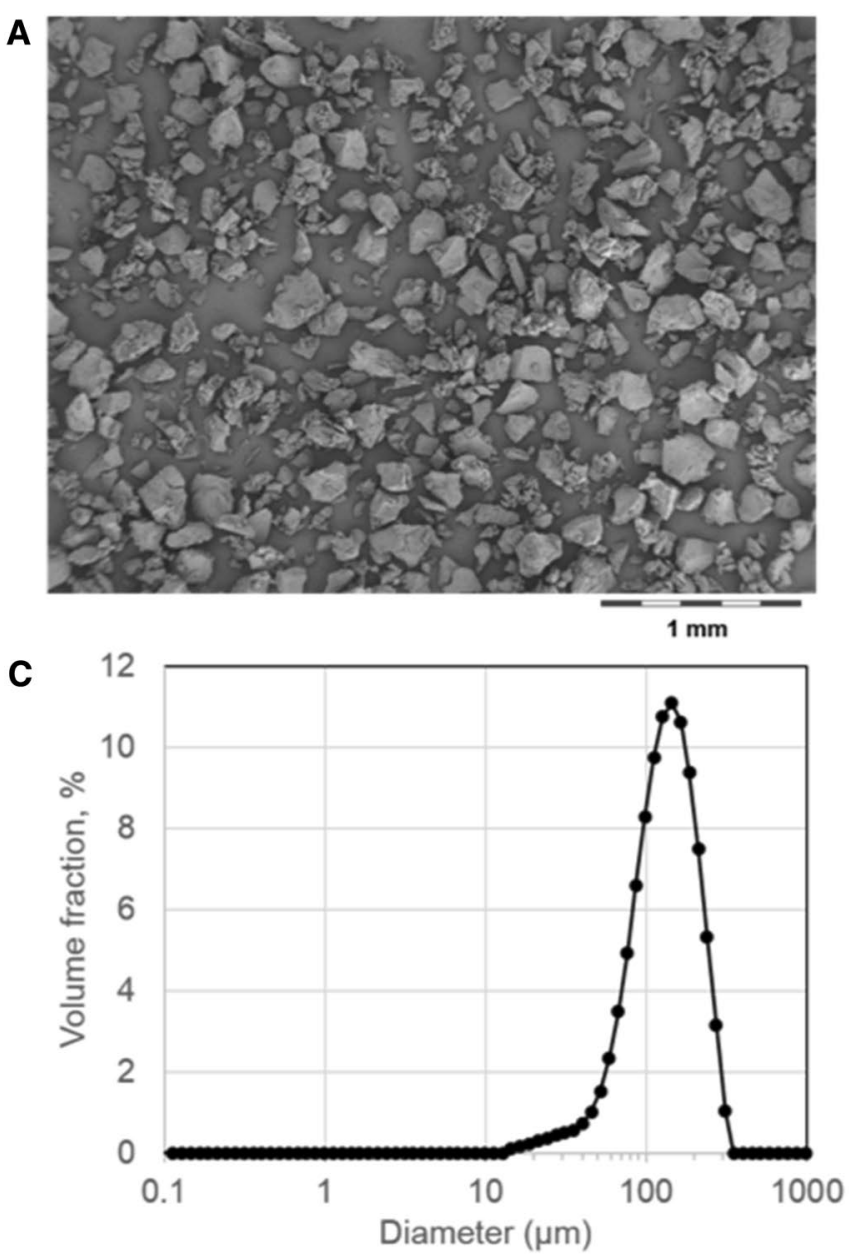

B

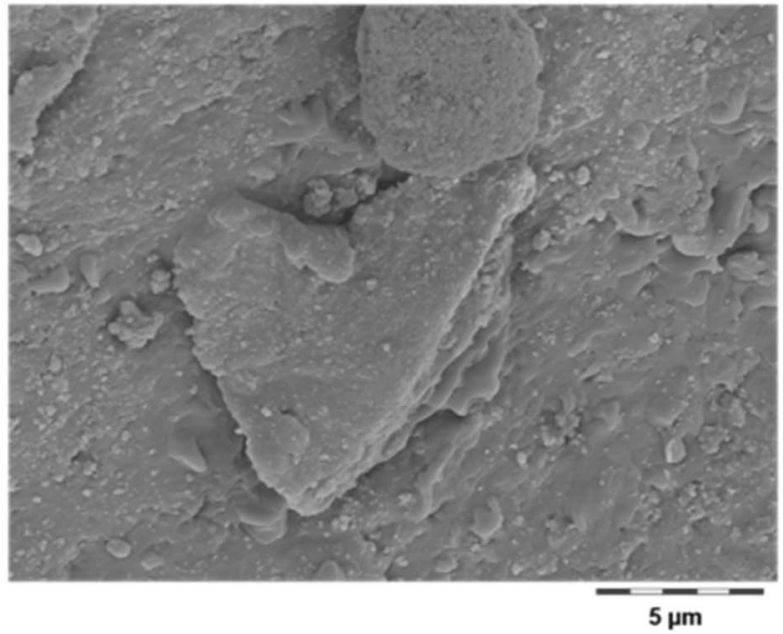

D
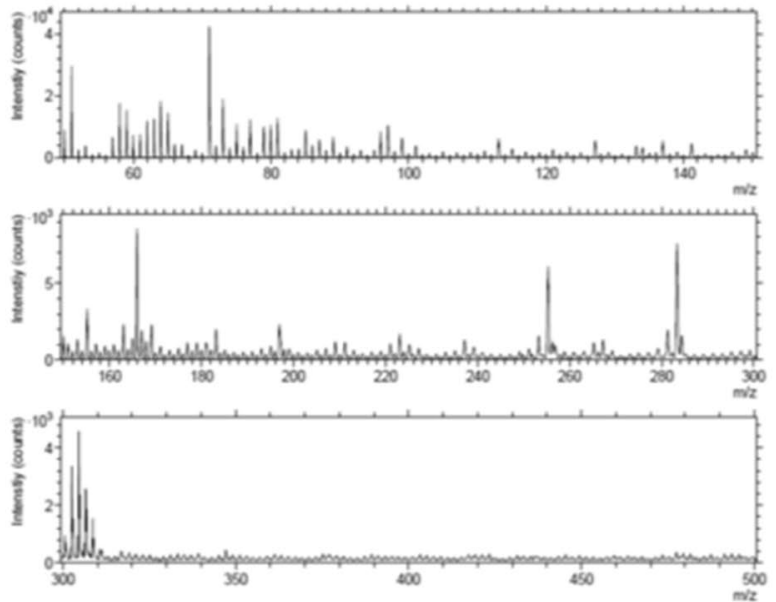

Fig. 1 Physical-chemical characterization of tire rubber. a, b SEM morphology (intermediate magnifications in Figure SI_6); $\mathbf{c}$ size distribution by laser diffraction; $\mathbf{d}$ surface chemistry by SIMS. Analogous results for all other test materials in Figure SI_1-SI_7

Table 1 Size distribution of test materials

\begin{tabular}{lcccl}
\hline Material & D10/ $\mu \mathrm{m}$ & $\mathrm{D} 50 / \mu \mathrm{m}$ & $\mathrm{D} 90 / \mu \mathrm{m}$ & Density $/ \mathrm{g} / \mathrm{cm}^{3}$ \\
\hline Healing earth & 3 & 26 & 55 & 2.65 \\
PA6 & 41 & 72 & 111 & 1.14 \\
Tire_rubber & 72 & 141 & 238 & $\mathrm{n} / \mathrm{a}$ \\
PU_hardened & 122 & 253 & 422 & 1.15 \\
TPU_ester & 152 & 264 & 432 & 1.19 \\
PP_Sun & 165 & 282 & 447 & 0.95 \\
\hline
\end{tabular}

identified. TPU is used, e.g., for shoe soles, and thus may generate wear particles. The micronized particles of TPU, PU-hardened, and PP have nearly identical size distributions (Figure SI_1). The particle shape is also comparable, although the PU_hardened has sharper edges on the micronized particles (Figure SI_4). The surface of PU_hardened bears some silicone contamination, which is absent in the bulk of the particles. The "healing earth", a kaolinite mineral, was sourced from the drugstore and was chosen as an inorganic control material that is intended for human oral consumption. Our results of microplastic particles on the order of $100 \mu \mathrm{m}$ (Figure SI_1) are consistent with earlier findings that a reduction of the sieve nominal mesh size to $100 \mu \mathrm{m}$ drastically reduces the passage down to $0.5 \%$ (Nowack et al. 2016). Polymer particles with sizes below $1 \mu \mathrm{m}$ or on the nanoscale may have an increased bioavailability, but only synergistic stresses by chemical degradation of the polymer and mechanical disruption of fragments can induce the secondary fragmentation of particles, as shown specifically for tire wear particles (Wohlleben et al. 2016) and PP pellets (Neubauer et al. 2017). The secondary fragmentation will scale with the chemical resilience of the matrix against chain scission, oxidation, and other forms of degradation by UV irradiation, hydrolysis, etc. Thus, if smaller particles are to be studied, they should likely consider a degraded form of the polymers. This is beyond the scope of the present contribution. 


\section{Development of the 3D co-culture model} and identification of mucus

In this study, we developed a three-dimensional intestinal in vitro co-culture model to assess inflammatory end points upon exposure to microplastic particles. The co-culture is composed of epithelial Caco-2 cells and mucus-secreting goblet cells HT29-MTX at a ratio of 9:1 on the upper side of the insert, combined with MDMs and MDDCs at the lower side at a ratio of 1:4. The cells in the co-culture setup were labeled with different vibrant dyes and subsequent imaging with LSM allowed for visualization of the spatial arrangement of the cells. The labeling showed monolayer formation of the enterocytes and a homogeneous distribution pattern of MDMs and MDDCs on the basolateral side of the insert (Fig. 2b). The formation of the microvilli brush boarder on the Caco-2 enterocytes was monitored by SEM, and indicated an increase of microvilli formation with increasing time (Fig. 2c).

The maturation state of MDMs and MDDCs was characterized by the visualization of the expression of the specific cell surface markers CD68 and CD83 (Fig. 3). Threedimensional reconstructions of the confocal data as well as immunofluorescence staining showed the specific surface antigen expression by the MDMs (CD68) and MDDCs
(CD83) in the co-culture system. Possible interactions of the immune cells with the epithelial monolayer were also investigated. For visualization of the cells, their actin-containing cytoskeleton was stained with Rhodamine-labeled phalloidin. Dendritic cells attached to the basolateral side of the insert were observed to form protrusions allowing for interaction with the epithelial monolayer as well as the apical compartment containing the microplastic particles.

Alcian blue staining was done to detect acidic mucus components in the different cultures. The surface of the HT29-MTX as well as the Caco-2/HT29-MTX co-cultures showed a clear blue coloring, suggesting the presence of mucus over the whole cell layer (Fig. 4). In comparison, the non-secreting mucin cell lines, such as the Caco- 2 cells as well as the human alveolar adenocarcinoma cell line A549, showed a very faint coloring. Light microscopy of paraffin-embedded vertical cross sections of the different cultures after day 21 using Alcian blue and eosin revealed a morphologically intact and continuous monolayer for the mono- and the co-cultures as well as the presence of mucin in the cultures containing HT29-MTX cells. In addition, $\mathrm{z}$-scans done with fluorescence microscopy of the Caco-2 monolayer and the Caco-2/HT29-MTX co-culture showed the expression of mucin by the goblet cells.

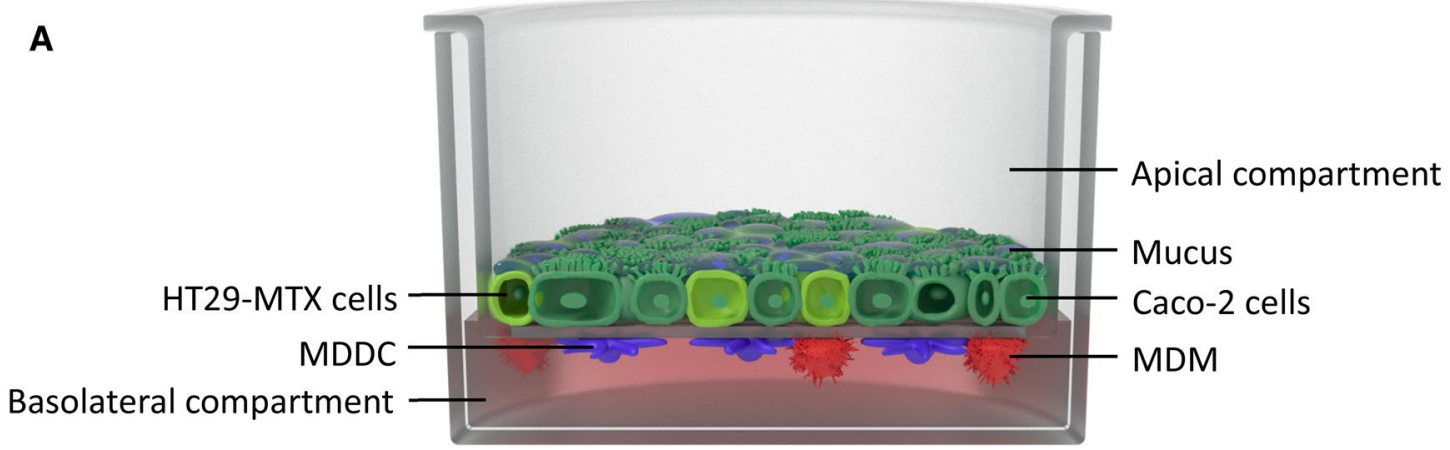

B

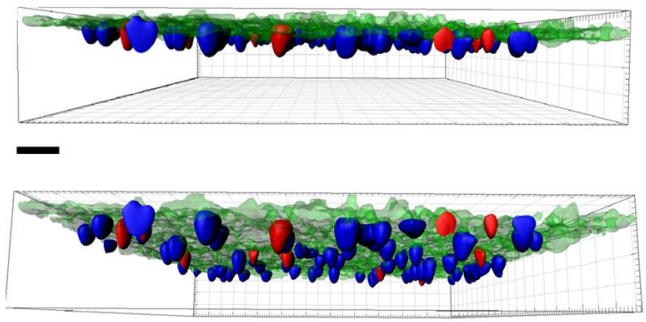

Fig. 2 a Schematic setup of the 3D co-culture model consisting of intestinal enterocytes (Caco-2) and mucus-producing HT29MTX cells, MDMs and MDDCs. b 3D visualization of the co-culture model showing Caco-2 monolayer (green), MDDCs (blue) and
C
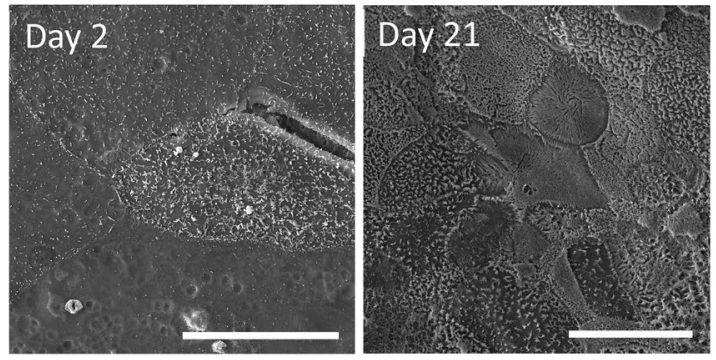

MDMs (red); scale bar represents $50 \mu \mathrm{m}$. c Determination of the microvilli brush boarder formation within 21 days by SEM. The SEM image shows the co-culture from the apical side of the insert. Scale bar $20 \mu \mathrm{m}$ (color figure online) 
A
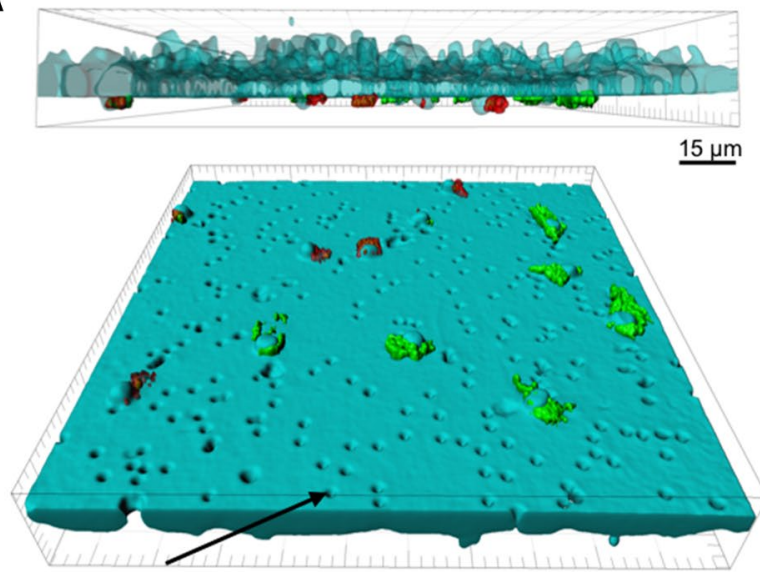

Pores of the insert
B
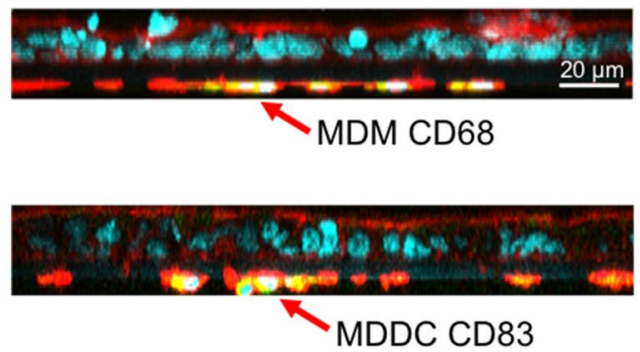

cyan $=$ cell nuclei red $=$ actin filament yellow/green $=$ immune cell

\section{C}

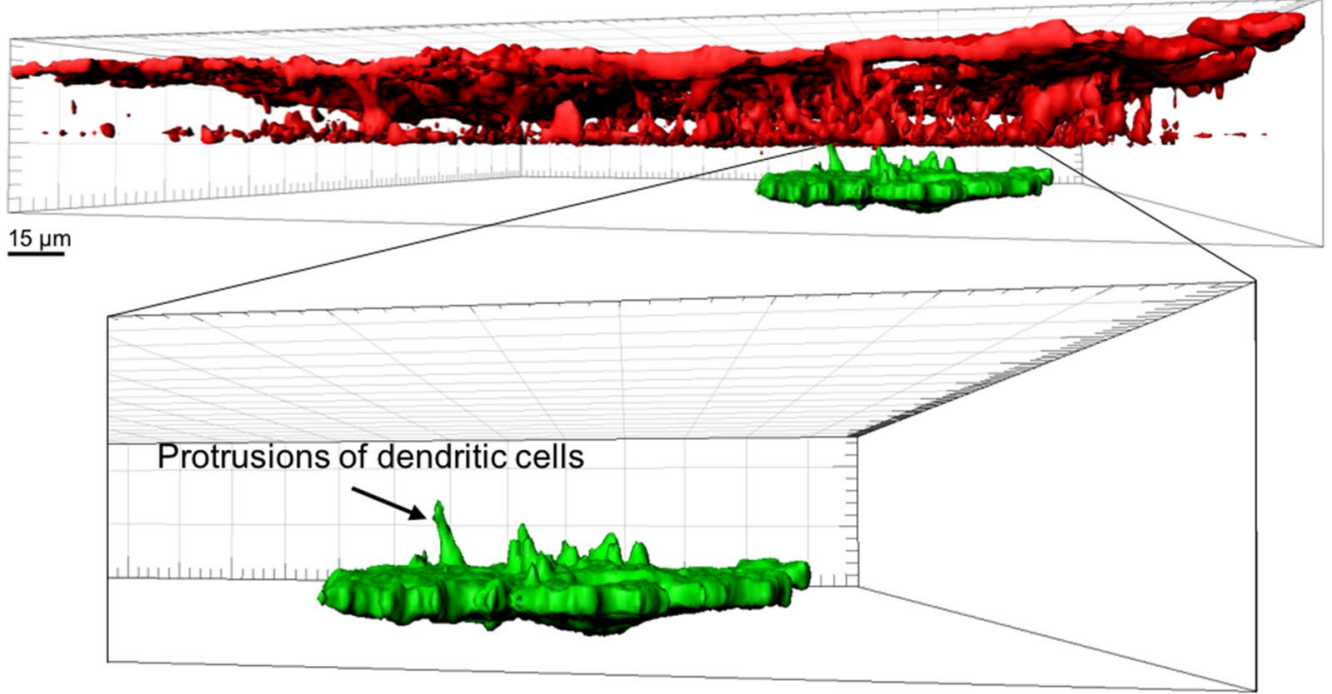

Fig. 3 Surface marker expression of macrophages and dendritic cells in co-culture (a, b) and protrusion formation of dendritic cells (c). Cells were fixed and stained for the specific surface markers CD68 and CD83. a 3D visualization of the co-culture showing Caco-2 monolayer/insert (cyan), MDMs CD68 (red) and MDDCs CD83 (green).

\section{Immunostaining of tight junction protein ZO-1}

The formation of the epithelial tissue barrier by tight junction (TJ) proteins to restrict the transport of ions, proteins, and other molecules is a crucial property of epithelial cells. The tight junction protein zonula occludens 1 (ZO-1) was labeled using fluorescently labeled antibodies. Using confocal microscopy, the immunostaining revealed the presence of ZO-1 proteins after day 21 toward the apical side of the epithelial layer for both the monoculture and the co-culture (Fig. 5). The Caco-2 monolayer revealed a homogeneously spread pattern of the TJs, whereas in the Caco-2/HT29MTX co-culture at the ratio 9:1, the pattern was interrupted because goblet cells form less TJs. In addition, areas with a b In addition, confocal laser scanning microscopy images showing cell-specific surface marker expression CD68 for MDM and CD83 for MDDCs. c 3D visualization of the actin filaments of Caco-2/ HT29-MTX co-culture (red) and MDDCs (green) showing the formation of protrusions (c) (color figure online)

strong fluorescence signal can be seen, which were attributed to an accumulation of the antibody in the mucus.

\section{Cell barrier integrity}

TEER measurements as well as permeability studies were conducted to assess the cell barrier integrity. An increased number of HT29-MTX cells resulted in decreased TEER values, as expected, due to the fact that HT29-MTX cells form less TJs (Walter et al. 1996). As mentioned in the literature, only cell cultures with TEER values above $200 \Omega \mathrm{cm}^{2}$ should be considered for experimental cultures. Cultures composed of Caco-2/HT29-MTX seeded at a ratio of 9:1 
Fig. 6 Monolayer integrity of Caco-2 and Caco-2/HT29-MTX co-cultures (a) TEER values. Different ratios of Caco-2/ HT29-MTX cells revealed TEER values above $200 \Omega \mathrm{cm}^{2}$ at a ratio of $9: 1$. b Permeability to $1 \mathrm{mg} / \mathrm{ml}$ fluorescein and $4 \mathrm{kDa}$ FITC dextran was monitored for $1 \mathrm{~h}$. Addition of EDTA induced a significant increase in the permeability rate for both $4 \mathrm{kDa}$ FITC dextran and fluorescein. A total of three independent experiments $(n=3)$, consisting of three single replicates each, were performed. Statistically significant difference to the positive control is denoted by asterisks, where $* * *$ is $p<0.001$
A

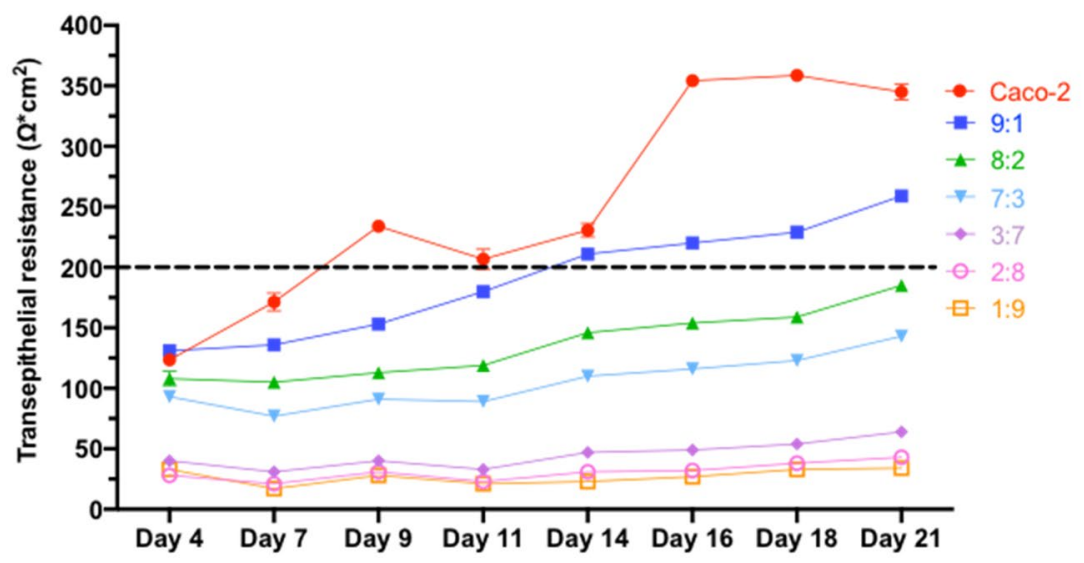

B 4kDa FITC Dextran

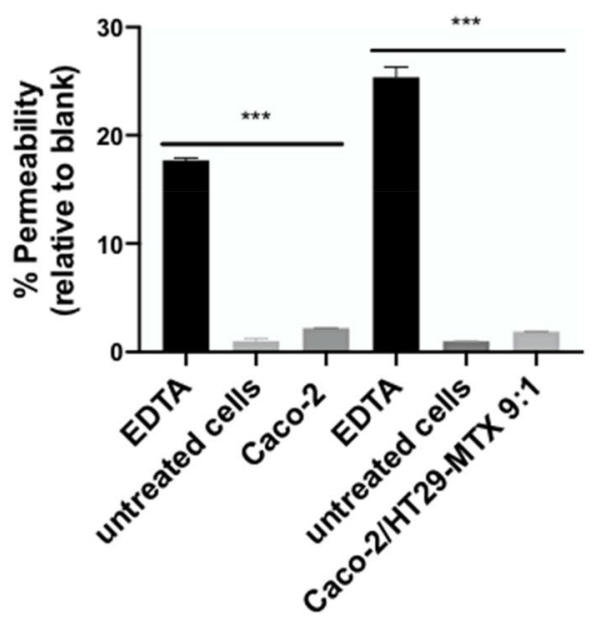

330Da Fluorescein

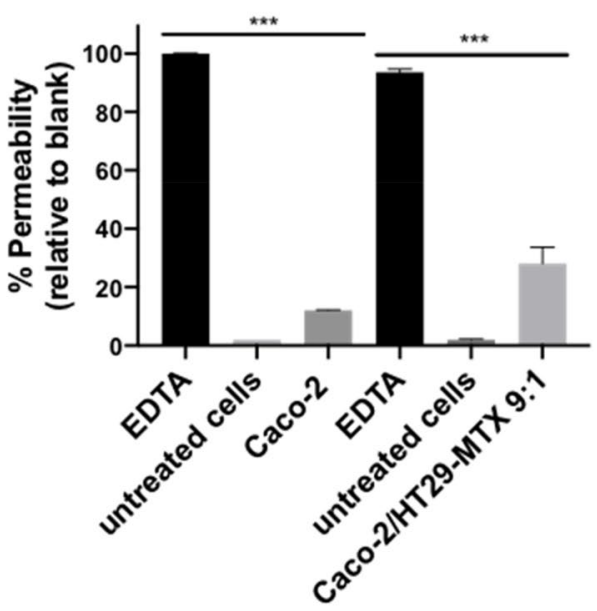

Table 2 Average delivered dose in $\mu \mathrm{g} / \mathrm{cm}^{2}$

\begin{tabular}{lll}
\hline Material exposed & Mean $\left(\mu \mathrm{g} / \mathrm{cm}^{2}\right)$ & SD \\
\hline Healing earth & 1101.83 & \pm 413.62 \\
PU_hardened & 1263.25 & \pm 592.80 \\
Tire rubber & 1066.00 & \pm 364.29 \\
PP_Sun & 1305.50 & \pm 474.80 \\
PA6 & 1028.58 & \pm 360.00 \\
TPU_ester & 1098.02 & \pm 312.18 \\
\hline
\end{tabular}

not induce any (pro-)inflammatory reactions during $48 \mathrm{~h}$ of exposure using doses up to $1380.0 \mu \mathrm{g} / \mathrm{cm}^{2}$.

The PP_Sun had no significant adverse effects up to the highest concentration tested in multiple ecotoxicity assays covering trout cell lines, microbial activity, and whole organisms (e.g.. the soil worm Enchytraeus crypticus and zebrafish embryo and larvae) (Amorim et al. 2018). Concerns were raised if such assays are relevant, considering that the biological entity (e.g,. cells and worms) are on the same size or even smaller than the test item (Scott-Fordsmand et al. 2017). However, this argument may not be valid for our differentiated co-culture that mimics tissue and approaches the in vivo situation, where the size of microparticles may indeed be an important parameter that prevents bioavailability. The effect on the barrier integrity after MP exposure was assessed by TEER measurements before and after 6, 24 and $48 \mathrm{~h}$. For all the materials, TEER values did not decrease after exposure (Fig. 9).

\section{Discussion}

In recent years, there is an increasing concern about microplastic particles in the environment, and emerging evidence suggests that human exposure, especially via contaminated food, can occur. However, the impacts of plastic in food is still unknown. Thus, we aimed to investigate the potential hazard posed by exposure of relevant microplastic particles to the human intestinal system by applying a novel 3D intestine tissue model. For this study, we chose polymers representing tire wear and polyolefins, which represent major sources of microplastic in the EU, and compared them to other polymer classes and an inorganic 

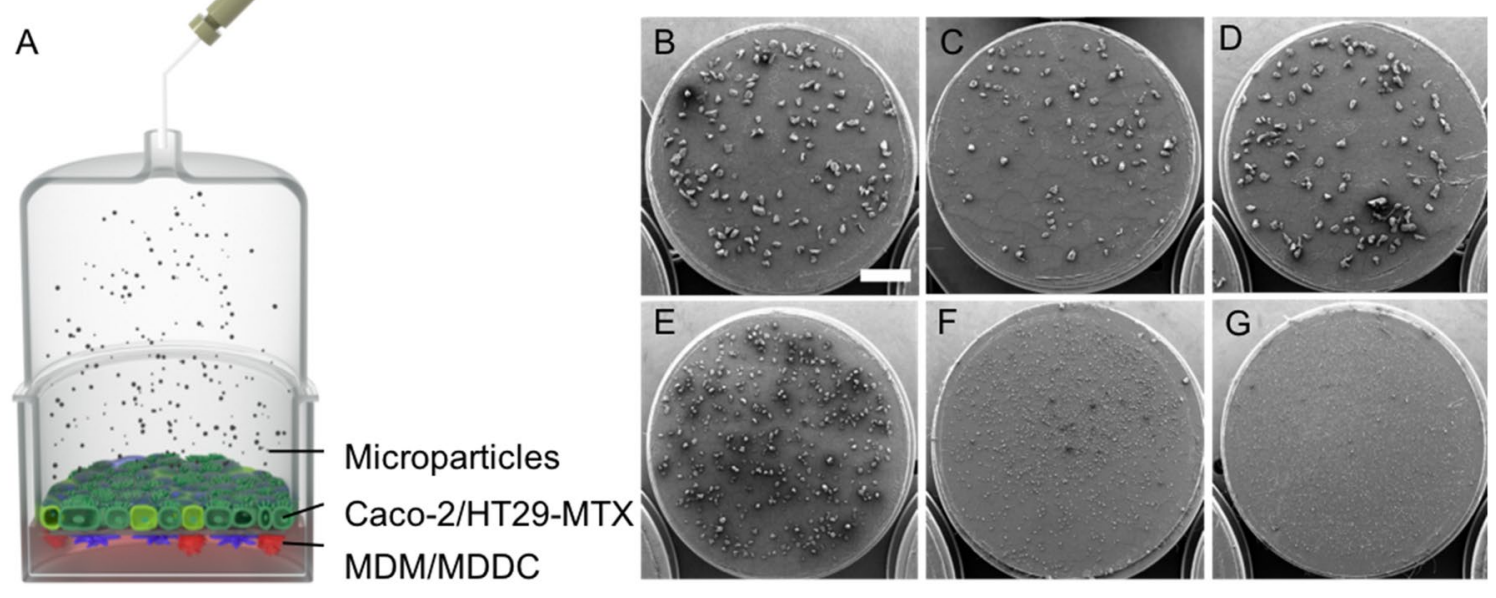

Fig. 7 a Nebulization outline for the exposure of the different microparticles, e.g.. microplastics and healing earth, to the co-culture system that included monocyte derived MDM and MDDC immune cells.

b-g Material exposure patterns imaged by SEM for PP_Sun (b), PU_ hardened (c), TPU_ester (d), tire rubber (e), PA6 (f) and healing earth (g). Scale bar $2 \mathrm{~mm}$

Fig. 8 Cytotoxicity determined by measuring the release of lactate dehydrogenase by the in vitro intestinal co-culture model after the exposure of microplastics and healing earth for 6,24 and $48 \mathrm{~h}$. A total of three independent experiments $(n=3)$, consisting of three single replicates each, were performed. Statistically significant difference to the positive control is denoted by asterisks, where $* * *$ is $p<0.001$

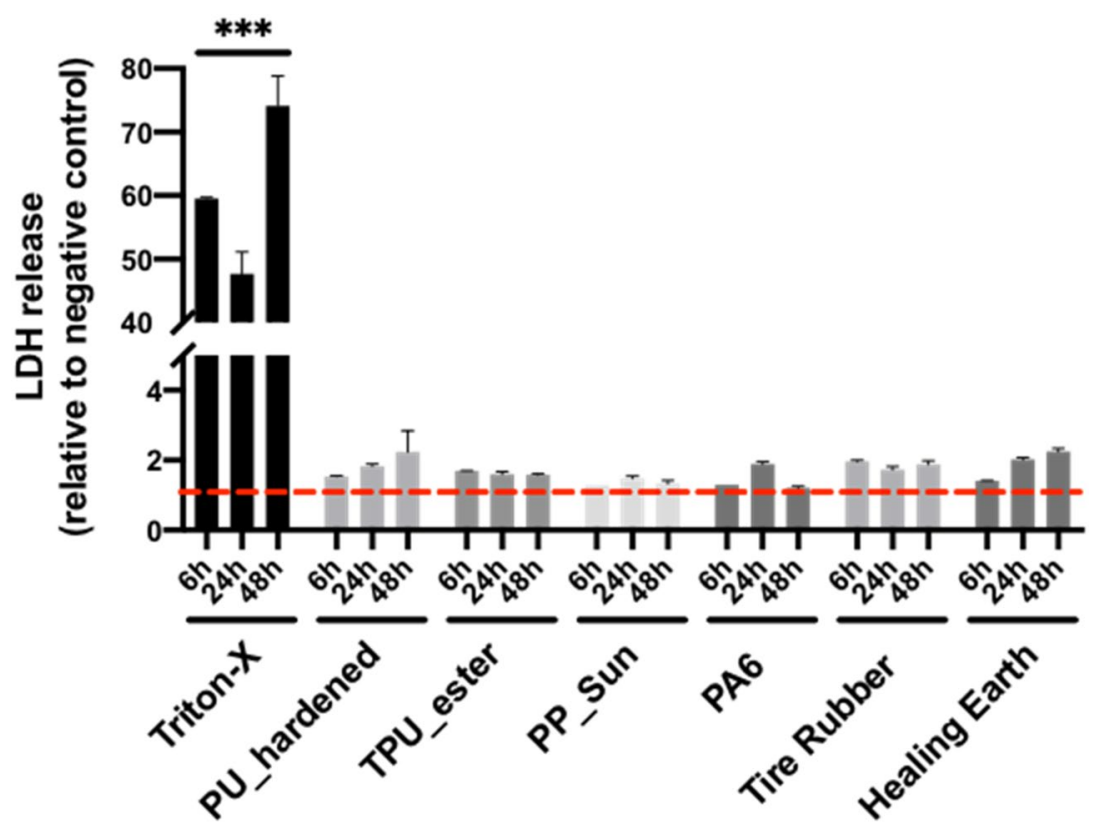

microparticle, healing earth, which is intended for human consumption. Powder from truck tires is commercially available. Truck tires consist of valuable natural rubber, filled with carbon black and other additives. After service life, they are recycled on a commercial scale by shredding and, optionally, by cryogenic micronization; the fine fractions of the recycled granulate are used for composite materials, while the coarse fractions are used for artificial turf. The polypropylene (PP), polyamide (PA6) and polyurethane (TPU) are all thermoplastics that are marketed in mm-sized pellets, and, as such, fulfill the proposed ECHA definition of microplastic. The PA6 is a generic thermoplastic grade that is not specifically intended for fishing nets, but is representative of microplastic fragments that 
focused on primary derived human monocytes as their behavior more closely resembles in vivo interactions and innate immune responses. We focused on Caco-2 and HT29MTX cells for the co-culture model. As the material we used was bigger than $70 \mu \mathrm{m}$, possible translocation across M-cells can be neglected. In our model we implemented the HT29MTX cell line, as mucus forms an important protective shield on the top of the epithelial layer and also reflects a more realistic in vitro scenario. In addition, regardless of the size of the particles exposed within this study, the membrane of the 12-well inserts used for our model, with pore sizes of $3 \mu \mathrm{m}$, do not allow the particles exposed to translocate across the epithelium to the basolateral side.

Polymers used for prosthetic materials, such as polyethylene, showed the formation of wear debris (Chen et al. 1995). These particles in the micron range can lead to a response by the innate immune system. Hallab et al. showed that $0.7,2$ and $10 \mu \mathrm{m}$ ultrahigh molecular weight polyethylene particles induced significant increases in IL-1 $\beta$, IL-6, IL- 8 and TNF $\alpha$ after $24 \mathrm{~h}$ of exposure to differentiated human macrophages (THP-1) as well as primary human monocytes (Hallab et al. 2012). To reveal possible interaction of the chosen materials with the immune system via the release of (pro-)inflammatory compounds, we introduced MDMs and MDDCs to the co-culture. Characterization of the expression of the surface specific cell markers CD68 and CD83 revealed the maturation of the immune cells. Furthermore, we could show the formation of protrusions by MDDCs allowing interactions with the intestine epithelium as also seen by studies from Rescigno and Borrow (2001) and Rimoldi et al. (2005).

Mucus production and identification of goblet cells within the co-culture were determined by histochemical analysis. The histological cross sections of the Caco-2 monolayer and the Caco-2/HT29-MTX co-culture stained with Alcian blue and eosin did not only show a confluent monolayer, but also indicated the presence of goblet cells as well the microvilli brush boarder. The latter was additionally confirmed by SEM images. In addition, the secretion of mucus was shown by immunostaining of mucin (MUC2). In Caco-2 monolayers, a thin layer of mucin was observed at the apical surface and it has already been shown by others that Caco- 2 cells are also able to express the MUC2 protein (Mattar et al. 2002). For the co-cultures, a more pronounced and less homogenous pattern could be observed as also described by Navabi et al. (2013). The integrity of the 3D model was further evaluated by $\mathrm{TJ}$ protein labeling and permeability investigations. ZO- 1 could be detected after day 21 toward the apical side of the epithelial layer for the Caco-2 monoculture as well as the co-culture. Contrary to the monocultures where a more homogenous TJ pattern was observed, the images of the Caco-2/HT29-MTX co-cultures show some interruptions because goblet cells form less TJs (Walter et al. 1996). This data lies in agreement with the TEER measurements, where a decrease in TEER with increased numbers of goblet cells was observed. In addition, the permeability assays with (FITC)-dextran $4 \mathrm{kDa}$ and fluorescein revealed a tight barrier formation for the monolayers and an increase in permeability of fluorescein in the co-culture that can be explained by the formation of fewer TJs, thus allowing smaller molecules to translocate through the layer.

To avoid the issue of preparing stable microplastic dispersions and to overcome the problem that many of the plastic particles are buoyant in water, we applied the particles directly in the bulk form using a dry powder insufflator to aerosolize the particles onto the cell surface. First, this represents the conditions in situ where the epithelial tissue is covered only by mucus but not liquid, and second, a homogenous material distribution over the whole inserts was demonstrated.

The only data available about reliable concentrations of microplastics to which humans might be exposed via the food chain are the studies of Van Cauwenberghe et al. and Li et al. Both studies investigated the amount of microplastics found in bivalves intended for consumption (Van Cauwenberghe and Janssen 2014; Li et al. 2015). Van Cauwenberghe examined mussels from a mussel farm in Germany as well as oysters bought in a supermarket originating from Brittany, France. For the mussels, an average of $0.36 \pm 0.07$ particles $/ g$ tissue and for the oysters $0.47 \pm 0.16$ particles/g tissue could be detected. Considering an average consumption of a portion of mussels, which is equal to about $250 \mathrm{~g}$, one consumes around 90 particles, whereas in the case of oysters, considering an average portion of 6 oysters, around 50 particles will be consumed. Li et al. investigated the amount of microplastics found in commercial bivalves from a fishery market in China. All the bivalves examined contained microplastics from 4.3 to 57.2 items/individual. Multiple types of microplastics were found in the tissue including fibers, fragments and pellets, and sizes less than $250 \mu \mathrm{m}$ were most frequent.

In our study, the concentrations were chosen according to the limitation of the insufflator system. On average the delivered dose that could be generated of all the materials was $1028.58-1305.5 \mu \mathrm{g} / \mathrm{cm}^{2}$ (Table 2). As seen by the exposure patterns of the materials (Fig. 7), the number of particles introduced at those doses are above 90 particles per insert. In addition, the size range was in the order of 50-500 $\mu \mathrm{m}$, which represents the mean range of microplastics found by $\mathrm{Li}$ et al. After considering that the whole food digestion process takes about $5 \mathrm{~h}$ to pass food from the stomach to the small intestine until it enters the large intestine for further digestion and absorption of water, we exposed the cells to the microplastics and healing earth particles for 6, 24 and $48 \mathrm{~h}$ (Kim et al. 1968). Due to limited knowledge about environmental concentrations and exposure concentrations via ingestion, it is not possible to hypothesize how realistic our exposure conditions are. 
In the present study, all the microplastics as well as the healing earth particles did not cause any significant cytotoxicity $(p<0.05)$ or release of (pro-)inflammatory cytokines (TNF $\alpha$, IL- 8 and IL-1 $\beta$ ). However, in the case of IL-8 a continuous increase in the release of the cytokine could be observed over $48 \mathrm{~h}$ for all the materials tested, which is not statistically significant in comparison to the negative control. In addition, a statistically significant effect was observed for all the tested positive controls proving the responsiveness of the employed 3D human intestinal model. Thermoplastics as well as cross-linked polymers are exempted from REACH registration, and thus only very limited data on the human and ecological hazard exist. Some of the polymer microparticles used in this study were found to be biologically inert against several aquatic species and fish cell lines (Amorim et al. 2018). This supports the absence of cytotoxicity. A recent study conducting a long-term exposure of up to 5 weeks, feeding $5 \mu \mathrm{m}$ fluorescent polystyrene beads at concentrations of $1000 \mu \mathrm{g} / \mathrm{l}$ to mice, revealed gut microbiota dysbiosis, intestinal barrier dysfunction, and metabolic disorders (Jin et al. 2019). As, so far, there are no data available about realistic concentrations of microplastics that might be ingested, extrapolation of these results to humans is critical and needs first experimental approval. Additional important points that also need to be considered are the possible impact of the various complex environments which particles will encounter in the digestive process (i.e., saliva, gastrointestinal fluids) and the impact of the mechanical forces present during digestion. The potential impact of digestive fluids on the particles would include changes to size, particle surface changes, and leaching of chemical compounds from the particles.

\section{Conclusions and outlook}

The biological response of a differentiated human intestine co-culture model was studied using the smallest microplastic fraction that can be generated by mechanical forces. The polymers represented tire wear and polyolefins, which represent major sources of microplastic in the EU, and they were compared against other polymer classes and an inorganic microparticle, healing earth, which is intended for human consumption. We designed a 3D intestine epithelial model to investigate the potential immune response upon exposure to the particles and found no induction of cytotoxicity nor (pro-)inflammatory response. The size of the microparticles studied here (on the order of 50-500 $\mu \mathrm{m}$ ) may contribute to the absence of a biological response, and particles of different size may induce effects. However, the present particles are the smallest size that could be attained by mechanical fragmentation-even smaller particles would originate only after microbiological or chemical degradation, which in turn reduces the persistence in the environment. The exposure of the cells to the microplastic via aerosolization allowed a homogenous deposition directly onto the cell surface. It was, however, not possible to investigate the interaction of particles with the cells, since the polymers got washed away from the inserts while they were being prepared for further staining experiments. Additionally, there are currently no data available about realistic concentrations of microplastics that might be ingested nor are there data on the possible effect of the complex environments present within the whole ingestion pathway (i.e., saliva and gastrointestinal fluids) on microplastic particle behavior. However, as this is the first study trying to assess the possible effects of microplastics on human health, extrapolation of these results to humans is critical. Further research will be required to determine the effects of smaller plastic fragments, and also chronic exposure of the particles to the intestine barrier should be considered. Possible scenarios may mimic daily food intake, including three to four exposures of the polymer particles per day.

Acknowledgements This work was supported by the Adolphe Merkle Foundation and BASF SE. We thank Dr. Miguel Spuch for the illustrational work of Figs. 2a and 6a and Prof. B. Schwaller from the Institute of Anatomy of the University Fribourg for the support with the histology cuts. R.L acknowledges funding from SPARK by Swiss National Science Foundation (190287). D.S. acknowledges funding from SPARK by Swiss National Science Foundation (190440).

\section{Compliance with ethical standards}

Conflict of interest RL and WW are employees of BASF SE, a company producing plastics.

\section{References}

Allen S, Allen D, Phoenix VR, Le Roux G (2019) Atmospheric transport and deposition of microplastics in a remote mountain catchment. Nat Geosci 12:339-344

Amorim MJB, Lin S, Schlich K et al (2018) Environmental impacts by fragments released from nanoenabled products: a multiassay, multimaterial exploration by the SUN approach. Environ Sci Technol 52:1514-1524. https://doi.org/10.1021/acs.est.7b04122

Antunes F, Andrade F, Araújo F et al (2013) Establishment of a triple co-culture in vitro cell models to study intestinal absorption of peptide drugs. Eur J Pharm Biopharm 83:427-435. https://doi. org/10.1016/j.ejpb.2012.10.003

Araújo F, Sarmento B (2013) Towards the characterization of an in vitro triple co-culture intestine cell model for permeability studies. Int J Pharm 458:128-134. https://doi.org/10.1016/j.ijpha rm.2013.10.003

Béduneau A, Tempesta C, Fimbel S et al (2014) A tunable Caco-2/ HT29-MTX co-culture model mimicking variable permeabilities of the human intestine obtained by an original seeding procedure. Eur J Pharm Biopharm 87:290-298. https://doi.org/10.1016/j. ejpb.2014.03.017

Bouwmeester H, Hollman PCH, Peters RJB (2015) Potential health impact of environmentally released micro- and nanoplastics in the 
human food production chain: experiences from nanotoxicology. Environ Sci Technol 49:8932-8947. https://doi.org/10.1021/acs. est. 5 b01090

Calatayud M, Vázquez M, Devesa V, Vélez D (2012) In vitro study of intestinal transport of inorganic and methylated arsenic species by Caco-2/HT29-MTX cocultures. Chem Res Toxicol 25:2654-2662. https://doi.org/10.1021/tx300295n

Chen PC, Mead EH, Pinto JG, Colwell CW (1995) Polyethylene wear debris in modular acetabular prostheses. Clin Orthop Relat Res 20:44-56

Cole M, Lindeque P, Halsband C, Galloway TS (2011) Microplastics as contaminants in the marine environment: a review. Mar Pollut Bull 62:2588-2597. https://doi.org/10.1016/j.marpo lbul.2011.09.025

ECHA (2019). https://echa.europa.eu/de/-/echa-proposes-to-restrictintentionally-added-microplastics

Edmondson R, Broglie JJ, Adcock AF, Yang L (2014) Three-dimensional cell culture systems and their applications in drug discovery and cell-based biosensors. Assay Drug Dev Technol 12:207-218. https://doi.org/10.1089/adt.2014.573

Eubeler JP, Bernhard M, Knepper TP (2010) Environmental biodegradation of synthetic polymers II. Biodegradation of different polymer groups. Trends Anal Chem 29:84-100. https://doi. org/10.1016/j.trac.2009.09.005

Fendall LS, Sewell MA (2009) Contributing to marine pollution by washing your face: microplastics in facial cleansers. Mar Pollut Bull 58:1225-1228. https://doi.org/10.1016/j.marpo lbul.2009.04.025

Hallab NJ, McAllister K, Brady M, Jarman-Smith M (2012) Macrophage reactivity to different polymers demonstrates particle size- and material-specific reactivity: PEEK-OPTIMA(®) particles versus UHMWPE particles in the submicron, micron, and 10 micron size ranges. J Biomed Mater Res Part B Appl Biomater 100:480-492. https://doi.org/10.1002/jbm.b.31974

Hilgendorf C, Spahn-Langguth H, Regårdh CG et al (2000) Caco-2 versus Caco-2/HT29-MTX co-cultured cell lines: permeabilities via diffusion, inside- and outside-directed carrier-mediated transport. J Pharm Sci 89:63-75. https://doi.org/10.1002/(SICI)15206017(200001)89:1\%3c63:AID-JPS7\%3e3.0.CO;2-6

Iñiguez ME, Conesa JA, Fullana A (2017) Microplastics in Spanish table salt. Sci Rep 7:8620. https://doi.org/10.1038/s41598-01709128-x

Jin Y, Lu L, Tu W et al (2019) Impacts of polystyrene microplastic on the gut barrier, microbiota and metabolism of mice. Sci Total Environ 649:308-317. https://doi.org/10.1016/j.scito tenv.2018.08.353

Kämpfer AAM, Urbán P, Gioria S et al (2017) Development of an in vitro co-culture model to mimic the human intestine in healthy and diseased state. Toxicol In Vitro 45:31-43. https://doi. org/10.1016/j.tiv.2017.08.011

Kim SK (1968) Small intestine transit time in the normal small bowel study. Am J Roentgenol Radium Ther Nucl Med 104:522-524

Kole PJ, Lohr AJ, Van Belleghem FGAJ, Ragas AMJ (2017) Wear and tear of tyres: a stealthy source of microplastics in the environment. Int J Environ Res Public Health 14:1265. https://doi.org/10.3390/ ijerph14101265

Kosuth M, Mason SA, Wattenberg EV (2018) Anthropogenic contamination of tap water, beer, and sea salt. PLoS ONE 13:e0194970. https://doi.org/10.1371/journal.pone.0194970

Lee J, Cuddihy MJ, Kotov NA (2008) Three-dimensional cell culture matrices: state of the art. Tissue Eng Part B Rev 14:61-86. https ://doi.org/10.1089/teb.2007.0150

Lehmann AD, Daum N, Bur M et al (2011) An in vitro triple cell coculture model with primary cells mimicking the human alveolar epithelial barrier. Eur J Pharm Biopharm 77:398-406. https://doi. org/10.1016/j.ejpb.2010.10.014
Leonard F, Collnot E-M, Lehr C-M (2010) A three-dimensional coculture of enterocytes, monocytes and dendritic cells to model inflamed intestinal mucosa in vitro. Mol Pharm 7:2103-2119. https://doi.org/10.1021/mp1000795

Li J, Yang D, Li L et al (2015) Microplastics in commercial bivalves from China. Environ Pollut 207:190-195. https://doi. org/10.1016/j.envpol.2015.09.018

Liebezeit G, Liebezeit E (2013) Non-pollen particulates in honey and sugar. Food Addit Contam Part A Chem Anal Control Expo Risk Assess 30:2136-2140. https://doi.org/10.1080/19440 049.2013 .843025

Liebezeit G, Liebezeit E (2014) Synthetic particles as contaminants in German beers. Food Addit Contam Part A Chem Anal Control Expo Risk Assess 31:1574-1578. https://doi.org/10.1080/19440 049.2014.945099

Mabbott NA, Donaldson DS, Ohno H et al (2013) Microfold (M) cells: important immunosurveillance posts in the intestinal epithelium. Mucosal Immunol 6:666-677. https://doi.org/10.1038/mi.2013.30

Mahler GJ, Esch MB, Tako E et al (2012) Oral exposure to polystyrene nanoparticles affects iron absorption. Nat Nanotechnol 7:264-271. https://doi.org/10.1038/nnano.2012.3

Mason SA, Welch VG, Neratko J (2018) Synthetic polymer contamination in bottled water. Front Chem 6:10377. https://doi. org/10.3389/fchem.2018.00407

Mattar AF, Teitelbaum DH, Drongowski RA et al (2002) Probiotics up-regulate MUC-2 mucin gene expression in a Caco-2 cell-culture model. Pediatr Surg Int 18:586-590. https://doi.org/10.1007/ s00383-002-0855-7

Navabi N, McGuckin MA, Lindén SK (2013) Gastrointestinal cell lines form polarized epithelia with an adherent mucus layer when cultured in semi-wet interfaces with mechanical stimulation. PLoS ONE 8:e68761. https://doi.org/10.1371/journal.pone.0068761

Neubauer N, Scifo L, Navratilova J et al (2017) Nanoscale coloristic pigments: upper limits on releases from pigmented plastic during environmental aging, in food contact, and by leaching. Environ Sci Technol 51:11669-11680. https://doi.org/10.1021/acs. est. $7 \mathrm{~b} 02578$

Nowack B, Boldrin A, Caballero A et al (2016) Meeting the needs for released nanomaterials required for further testing-the SUN approach. Environ Sci Technol 50:2747-2753. https://doi. org/10.1021/acs.est.5b04472

Pinto M, Robineleon S, Appay MD et al (1983) Enterocyte-like differentiation and polarization of the human-colon carcinoma cell-line Caco-2 in culture. Biol Cell 47:323-330

Plastic Europe (2018) Plastics - the Facts 2017. PlasticEurope 1-44.

Prata JC (2018) Airborne microplastics: consequences to human health? Environ Pollut 234:115-126. https://doi.org/10.1016/j. envpol.2017.11.043

Rescigno M, Borrow P (2001) The host-pathogen interaction: new themes from dendritic cell biology. Cell 106:267-270

Rezania S, Park J, Md Din MF et al (2018) Microplastics pollution in different aquatic environments and biota: a review of recent studies. Mar Pollut Bull 133:191-208. https://doi.org/10.1016/j. marpolbul.2018.05.022

Rimoldi M, Chieppa M, Larghi P et al (2005) Monocyte-derived dendritic cells activated by bacteria or by bacteria-stimulated epithelial cells are functionally different. Blood 106:2818-2826. https ://doi.org/10.1182/blood-2004-11-4321

Rochman CM, Tahir A, Williams SL et al (2015) Anthropogenic debris in seafood: plastic debris and fibers from textiles in fish and bivalves sold for human consumption. Sci Rep 5:14340. https ://doi.org/10.1038/srep14340

Ryan PG, Moore CJ, van Franeker JA, Moloney CL (2009) Monitoring the abundance of plastic debris in the marine environment. Philos Trans R Soc Lond B Biol Sci 364:1999-2012. https://doi. org/10.1098/rstb.2008.0207 
Schimpel C, Teubl B, Absenger M et al (2014) Development of an advanced intestinal in vitro triple culture permeability model to study transport of nanoparticles. Mol Pharm 11:808-818. https:// doi.org/10.1021/mp400507g

Scott-Fordsmand JJ, Navas JM, Hund-Rinke K et al (2017) Nanomaterials to microplastics: Swings and roundabouts. Nano Today 17:7-10. https://doi.org/10.1016/j.nantod.2017.09.002

Steffens KJ (1995) Persorption-criticism and agreement as based upon in vitro and in vivo studies on mammals. Absorption of orally administered enzymes. Springer, Berlin

Susewind J, de Souza C-W, Repnik U et al (2016) A 3D co-culture of three human cell lines to model the inflamed intestinal mucosa for safety testing of nanomaterials. Nanotoxicology 10:53-62. https ://doi.org/10.3109/17435390.2015.1008065

Van Cauwenberghe L, Janssen CR (2014) Microplastics in bivalves cultured for human consumption. Environ Pollut 193:65-70. https ://doi.org/10.1016/j.envpol.2014.06.010

Walter E, Janich S, Roessler BJ et al (1996) HT29-MTX/Caco-2 cocultures as an in vitro model for the intestinal epithelium: in vitro-in vivo correlation with permeability data from rats and humans. J Pharm Sci 85:1070-1076. https://doi.org/10.1021/js960110x

Wohlleben W, Meyer J, Muller J et al (2016) Release from nanomaterials during their use phase: combined mechanical and chemical

stresses applied to simple and multi-filler nanocomposites mimicking wear of nano-reinforced tires. Environ Sci Nano 3:10361051. https://doi.org/10.1039/C6EN00094K

World Health Organization (2019) Microplastics in drinking-water

Wright SL, Kelly FJ (2017) Plastic and human health: a micro issue? Environ Sci Technol 51:6634-6647. https://doi.org/10.1021/acs. est.7b00423

Wu WM, Yang J (2017) Microplastics pollution and reduction strategies. Criddle Front Environ Sci. https://doi.org/10.1007/s1178 3-017-0897-7

Yousif E, Haddad R (2013) Photodegradation and photostabilization of polymers, especially polystyrene: review. Springerplus 2:398. https://doi.org/10.1186/2193-1801-2-398

Zietarska M, Maugard CM, Filali-Mouhim A et al (2007) Molecular description of a 3D in vitro model for the study of epithelial ovarian cancer (EOC). Mol Carcinog 46:872-885. https://doi. org/10.1002/mc.20315 